\title{
Factors Associated With Delay in Diagnosis and Treatment of Pulmonary Tuberculosis
}

\author{
Seyed Mohammad Alavi ${ }^{1, *} ;$ Pejman Bakhtiyariniya ${ }^{2}$; Ali Albagi $^{2}$ \\ ${ }_{1}^{1}$ Health Research Institute, Infectious and Tropical Disease Research Center, Ahvaz Jundishapur University of Medical Sciences, Ahvaz, IR Iran \\ 2 Khuzestan Health Center, Tuberculosis Department, Ahvaz Jundishapur University of Medical Sciences, Ahvaz, IR Iran \\ ${ }^{*}$ Corresponding author: Seyed Mohammad Alavi, Health Research Institute, Infectious and Tropical Disease Research Center, Ahvaz Jundishapur University of Medical Sciences, \\ Ahvaz, IR Iran. Tel: +98-6133387724, E-mail: alavi.seyedmohammad@yahoo.com, alavi-sm@ajums.ac.ir
}

Received: April 1, 2014; Revised: July 27, 2014; Accepted: August 27, 2014

\begin{abstract}
Background: Early treatment of pulmonary tuberculosis (PTB) is necessary for a successful tuberculosis(TB) control program. Objectives: The objective of this study was to determine total treatment delay and its associated factors among PTB patients in Ahvaz. Patients and Methods: A retrospective study was performed among newly diagnosed PTB cases registered in 2010 at the Ahvaz health center. Total treatment delay was defined as the time interval between the onsets of cough to the initiation of anti-TB treatment. Tuberculosis diagnosis and treatment was based on the national TB program (NTP). Data analysis was performed using the SPSS software by chi-square and Fisher's exact test with odds ratio (OR) and 95\% confidence interval(CI).

Results: The mean age of the patients was $38.9 \pm 12.3$ years; 83 were male and 56 were female. Of the 139 smear positive PTB cases, 91 ( $65.5 \%$ ) cases had received delayed-treatment. The mean time between onset of symptoms, diagnosis and treatment was 73 days (median: 48 days, range: 4-570 days). Female gender $(\mathrm{OR}(95 \% \mathrm{CI}): 2.9,1.03-8.23, \mathrm{P}=0.02)$, smoking $(\mathrm{OR}(95 \% \mathrm{CI}): 0.49,0.22-0.96, \mathrm{P}=0.04)$ and receiving immunosuppressive drugs (OR (95\% CI): 8.18, 1.09-75.31, $\mathrm{P}<0.05$ ) were associated with longer delayed time.

Conclusions: Delayed diagnosis and treatment of tuberculosis appears to be the main problem in the TB control program of the region. Delayed time is significantly associated with female gender, smoking and immunosuppressive drugs.
\end{abstract}

Keywords: Tuberculosis; Diagnosis; Treatment

\section{Background}

Although in the recent years, tuberculosis (TB) control has improved by the implementation of programs such as directly observed treatment short course (DOTS) and stop TB strategy yet, TB remains a public health problem in middle- and low-income countries $(1,2)$. Tuberculosis with two million deaths each year and incidence of approximately nine million, after human immunodeficiency virus (HIV), is second among lethal infectious agents in the world ranking $(2,3)$. More than $90 \%$ of TB cases occur in developing countries, and about 75\% happen in the $15-54$ age group. Absences at workplaces due to illness and TBrelated death resulted in about a 30\% family income reduction (4). Tuberculosis is a major public health problem in $\operatorname{Iran}(5)$.

Khuzestan province in southwestern Iran, due to geopolitical circumstances and having a long border with Iraq is very important in terms of TB control. Iranian Center for Diseases Control (CDC) (ministry of health) has classified Khuzestan in the mid zone of high burden provinces with TB cases. In a study conducted in Khuzestan, incidence of $\mathrm{TB}$ in this province was estimated to be 50 per 100000 individuals (6). Early diagnosis and appropriate treatment of TB are the most important indicators of disease control in the community (7). One of the reasons for the failure of
TB control is a delay in diagnosis and treatment (2). Delay in initiating treatment results in increasing severity of the disease, mortality and transmission. If the interval between the presentation of the first symptoms of disease, diagnosis and treatment of disease is prolonged, the risk of TB transmission will be increased.

With progression of pulmonary lesions, the likelihood of bacterial resistance and mortality is increased and consequently successful treatment outcome will be decreased (8). One of the serious problems of TB control in Khuzestan similar to other parts of Iran is the emergence of multidrug-resistant tuberculosis (MDR-TB). Poor treatment outcome of tuberculosis led to MDR-TB making the control of tuberculosis more complex and this vicious cycle continues (9). Delay in diagnosis and treatment of pulmonary tuberculosis (PTB) is one of the reasons for the failure to control the disease. Determination of the causes of delay in timely diagnosis and treatment is one of the most important steps that must be taken for implementing the national program for TB (NTP) in each region. Much of the delay in diagnosis and treatment of disease are due to two reasons, delays of patients in referring to health centers and delay by the health system (health care workers and health staff). The high treatment success rate

Copyright (C) 2015, Ahvaz Jundishapur University of Medical Sciences. This is an open-access article distributed under the terms of the Creative Commons Attribution-NonCommercial 4.0 International License (http://creativecommons.org/licenses/by-nc/4.0/) which permits copy and redistribute the material just in noncommercial usages, provided the original work is properly cited. 
of Directly Observed Treatment Short Course (DOTS) may be achieved by ways of shortening diagnostic delays (3). A few studies about the causes of delays in Iran are available (5) yet so far we have found no study on this topic that has been conducted in the Khuzestan province.

\section{Objectives}

Therefore, this study was conducted to investigate reasons of delay in TB diagnosis and treatment in this region.

\section{Patients and Methods}

In a cross-sectional study in the Ahvaz Health Center, newly diagnosed sputum smear positive patients with PTB in 2010 were investigated. Medical information of patients, from the time of first clinical presentation to the time of diagnosis based on sputum examination, and starting treatment were studied. Date of the first manifestation of PTB, smear positive diagnosis and initiation of anti-TB drug were extracted from the patient's files. Total treatment delay (TTD) was defined as the time interval between the onset of cough (more than two weeks of duration) to the first visit to private physician or health center, plus the health system delay, which is the interval from the first visit to the initiation of anti-TB treatment $(7,10)$. Total treatment delay of less than four weeks (time of the first symptom presentation to time of receiving drugs) was defined as timely treatment; TTD of more than four weeks was classified as delayed treatment.

Tuberculosis diagnosis and treatment was based on NTP.
Cases with at least two sputum smears positive for acid fast bacillus (SSP-AFB) or, a chest radiography suggestive of tuberculosis plus one SSP-AFB or, sputum culture positive for TB and one SSP-AFB were defined as sputum smear positive pulmonary tuberculosis. Patients with PTB were placed in two groups of early detected and treated (EDT) and late detected and treated (LDT). Data for each group, including age, gender, location, education, HIV infection, intravenous drug abuse, underlying disease, prison history, symptoms, medications and other variables related to the study were analyzed. Data analysis was performed using the SPSS software by use of chi-square and Fisher's exact test with odds ratio (OR) and 95\% confidence interval (CI).

\section{Results}

This study included 181 PTB cases, amongst which 139 (76.8\%) were smear-positive. The mean age of the patients was $38.9 \pm 12.3$ years, 83 were male and 56 were female. Of the 139 smear positive PTB cases, 48 (34.5\%) patients were in the EDT group and 91 (65.5\%) cases were in the LDT group. Mean age of both groups was similar (34.1 years versus 34.9 years, $\mathrm{P}=0.54$ ). The mean time between onset of symptoms and diagnosis and treatment was 73 days (median: 48 days, range: 4-570 days). Frequency of factors affecting diagnosis and treatment of both groups are shown in Table 1. Female gender (OR (95\% CI): 2.9, 1.03-8.23, $\mathrm{P}=0.02)$, smoking (OR (95\% CI): 0.49, 0.22-0.96, $\mathrm{P}=0.04)$ and receiving immunosuppressive drugs (OR (95\% CI): 8.18, 1.09-75.31, P < 0.05) were associated with longer total delay.

\begin{tabular}{|c|c|c|c|c|}
\hline Factors & EDT Group $(n=48)$ & LDT Group $(\mathrm{n}=91)$ & Odds Ratio (95\% CI) & PValue \\
\hline \multicolumn{5}{|l|}{ Age, $y$} \\
\hline$<15$ & $1(2.1)$ & $3(3.3)$ & $0.62,0.06-6.17$ & 0.52 \\
\hline $15-65$ & $40(83.3)$ & $76(83.5)$ & $0.99,0.39-2.53$ & 0.57 \\
\hline$>65$ & $7(14.6)$ & $12(13.2)$ & $1.12,0.41-3.07$ & 0.50 \\
\hline Gender & & & $2.9,1.03-8.23$ & 0.02 \\
\hline Male & $43(89.6)$ & $68(74.7)$ & & \\
\hline Female $^{\mathrm{c}}$ & $5(10.4)$ & $23(25.3)$ & & \\
\hline Residency & & & & 0.09 \\
\hline Urban & $26(54.2)$ & $61(67.0)$ & $0.58,0.28-1.19$ & \\
\hline Rural & $22(45.8)$ & $30(33.0)$ & & \\
\hline Imprisonment & $7(14.6)$ & $13(14.3)$ & $1.02,0.38-2.77$ & 0.57 \\
\hline IVDU & $3(6.3)$ & $4(4.4)$ & $1.45,0.31-6.76$ & 0.45 \\
\hline HIV infection & $2(4.2)$ & $1(1.1)$ & $3.91,0.35-44.30$ & 0.27 \\
\hline Bronchial Asthma & $2(4.2)$ & $7(7.7)$ & $0.52,0.10-2.62$ & 0.34 \\
\hline COPD & $3(6.3)$ & $9(9.9)$ & $0.61,0.16-2.36$ & 0.35 \\
\hline Smoking ${ }^{\mathrm{C}}$ & $12(25.0)$ & $37(40.7)$ & $0.49,0.22-0.96$ & 0.04 \\
\hline Immunosuppressive drugs ${ }^{\mathrm{C}}$ & $4(8.3)$ & $1(1.1)$ & $8.18,1.09-75.31$ & $<0.05$ \\
\hline DM & $6(12.5)$ & $7(7.7)$ & $1.77,0.54-5.42$ & 0.26 \\
\hline
\end{tabular}


Alavi SM et al.

\section{Discussion}

Studies conducted in different countries have shown that many factors are involved in delayed diagnosis and treatment of tuberculosis (5, 7, 8, 10-15). Patient and health system delays in TB management are mediated by individual, socioeconomics and diagnostic factors. An important factor is the lack of sufficient awareness of signs and symptoms of TB in the general population or health workers (16). Low TB knowledge caused a delay in referring patients to facilities, as well as a delay in tuberculosis diagnostic health system (17). However, because of the retrospective nature of this study we could not evaluate patients' causes of delay when referring to health centers. However, other studies conducted in this region (18) have revealed that awareness about the disease in the general population and in some health professionals was low. The present study revealed that the median of total delay from the first TB symptom to initiating treatment was 73 days. In previous studies this delay ranged from 25 days to 185 days (3).

In a study by Nasehi et al. (5) this duration was 54 days, while Cheng et al.(15) reported 58 days, Hussen et al.(17) 97 days, and Saifodine et al. (11) 150 days. The reason for these differences depends on people's access to health services in various areas, (particularly in remote areas) quality of DOTS programs, public awareness of TB, people's level of education, socio-economic status and resource limitation in different areas $(1,3,13,16,19)$. In this study, $65.5 \%$ of patients started anti-tuberculosis treatment with a delay of more than four weeks. This long duration of delay in TB treatment happened in $70 \%$ of patients as reported by Saifodine et al. (11) in Mozambique and in $72.5 \%$ of patients by the study of Guneylioglu et al. (19) and colleagues in Turkey. As mentioned previously, socio-economic status, population distribution, the percentage of literate individuals, quality of health care services, and general poverty in different areas can explain these differences. There are many factors associated with delays in diagnosis and initiating treatment. These factors have not always been similar in all societies and even within countries $(2,3,16)$. Storla et al. (2) in a meta-analysis of factors on delays in TB diagnosis and treatment explained that HIV infection, being of old age, low level of education, long distance to health facilities, male gender, first visit by physician not familiar to NTP, rural residence and HIV infection were the most prevalent factors associated with diagnostic and treatment delays. In our study, several factors such as sex, old age, rural and urban settlements, debilitating diseases (such as diabetes, bronchial asthma and chronic obstructive pulmonary disease), smoking, intravenous drug abuse, HIV infection and history of incarceration were examined. Among these factors, female sex, drug addiction, asthma, chronic lung disease, smoking, immunosuppressive drug consumption and diabetes mellitus may be considered as factors influencing delay in treatment, although the difference was significant only in women, smokers and immunosuppressive drug consumers $(<0.05)$.

Our findings are consistent with that of Storla et al. (2) and other investigators. The reason for longer delays among patients living in urban areas compared to rural areas may be due to the fact that the Iranian health system is based on primary health care (PHC) and is mainly located in rural areas. Furthermore, HIV positive patients had less patient delay probably because of activities of consulting behavioral disease clinics (called triangle clinics) in Iranian health settings for HIV, drug addiction and sexually transmitted diseases (STD). The collaborative TB/ HIV program that exits in health settings and prisons ensures early TB detection among HIV patients and prisoners. Smokers neglect coughing for more than two weeks as the main symptom of pulmonary tuberculosis due to cigarette induced cough. Immunosuppressive drugs inhibit coughing as the main symptom of pulmonary tuberculosis. This study had some limitations. The study design was retrospective, therefore, it is possible that data related to patient's delay factors such as, socioeconomics, patients behavior and patients knowledge about TB were not recorded in the case notes. Another limitation was the lack of data about TB knowledge and attitudes of health care workers. Therefore, this study can be used as a basic study for future researches.

\section{Acknowledgements}

The authors wish to thank the chief and personnel of Infectious and Tropical Disease Research Center, Ahvaz Jundishapur University of Medical Sciences, Ahvaz, IR Iran for supporting this study as well as the chief and personnel of the Khuzestan Health Center for their warm cooperation.

\section{Authors' Contributions}

Study concept and design: Seyed Mohammad Alavi. Literature search: Seyed Mohammad Alavi. Drafting of the manuscript: Seyed Mohammad Alavi and Pejman Bakhtiyariniya. Critical revision of the manuscript for important intellectual content: Seyed Mohammad Alavi. Advising: Seyed Mohammad Alavi and Ali Albagi. Statistics tests: Pejman Bakhtiyariniya and Ali Albagi.

\section{Funding/Support}

This study was supported by the Infectious and Tropical Research Center of Ahvaz Jundishapur University of Medical Sciences; Ahvaz, IR Iran.

\section{References}

1. Deponti GN, Silva DR, Coelho AC, Muller AM, Dalcin Pde T. Delayed diagnosis and associated factors among new pulmonary tuberculosis patients diagnosed at the emergency department of a tertiary care hospital in Porto Alegre, South Brazil: a prospective patient recruitment study. BMC Infect Dis. 2013;13:538.

2. Storla DG, Yimer S, Bjune GA. A systematic review of delay in 
the diagnosis and treatment of tuberculosis. BMC Public Health. 2008;8:15.

3. Sreeramareddy CT, Panduru KV, Menten J, Van den Ende J. Time delays in diagnosis of pulmonary tuberculosis: a systematic review of literature. BMC Infect Dis. 2009;9:91.

4. Lin HP, Deng CY, Chou P. Diagnosis and treatment delay among pulmonary tuberculosis patients identified using the Taiwan reporting enquiry system, 2002-2006. BMC Public Health. 2009;9:55.

5. Nasehi M, Hassanzadeh J, Rezaianzadeh A, Zeigami B, Tabatabaee $\mathrm{H}$, Ghaderi E. Diagnosis delay in smear positive tuberculosis patients. J Res Med Sci. 2012;17(11):1001-4.

6. Alavi SM, Sefidgaran GH. Tuberculin survey among school-aged children in Ahvaz, Iran, 2006. Int J Infect Dis. 2008;12(4):406-9.

7. Yimer SA, Bjune GA, Holm-Hansen C. Time to first consultation, diagnosis and treatment of TB among patients attending a referral hospital in Northwest, Ethiopia. BMC Infect Dis. 2014;14:19.

8. Belay M, Bjune G, Ameni G, Abebe F. Diagnostic and treatment delay among Tuberculosis patients in Afar Region, Ethiopia: a cross-sectional study. BMC Public Health. 2012;12:369.

9. Nasiri MJ, Rezaei F, Zamani S, Darban-Sarokhalil D, Fooladi AA, Shojaei H, et al. Drug resistance pattern of Mycobacterium tuberculosis isolates from patients of five provinces of Iran. Asian Pac J Trop Med. 2014;7(3):193-6.

10. Makwakwa L, Sheu ML, Chiang CY, Lin SL, Chang PW. Patient and heath system delays in the diagnosis and treatment of new and retreatment pulmonary tuberculosis cases in Malawi. BMC Infect Dis. 2014;14:132.

11. Saifodine A, Gudo PS, Sidat M, Black J. Patient and health system delay among patients with pulmonary tuberculosis in Beira city,
Mozambique. BMC Public Health. 2013;13:559.

12. Chiang CY, Chang CT, Chang RE, Li CT, Huang RM. Patient and health system delays in the diagnosis and treatment of tuberculosis in Southern Taiwan. Int J Tuberc Lung Dis. 2005;9(9):1006-12.

13. Okur E, Yilmaz A, Saygi A, Selvi A, Sungun F, Ozturk E, et al. Patterns of delays in diagnosis amongst patients with smear-positive pulmonary tuberculosis at a teaching hospital in Turkey. Clin Microbiol Infect. 2006;12(1):90-2.

14. Zerbini E, Chirico MC, Salvadores B, Amigot B, Estrada S, Algorry G. Delay in tuberculosis diagnosis and treatment in four provinces of Argentina. Int J Tuberc Lung Dis. 2008;12(1):63-8.

15. Cheng S, Chen W, Yang Y, Chu P, Liu X, Zhao M, et al. Effect of Diagnostic and Treatment Delay on the Risk of Tuberculosis Transmission in Shenzhen, China: An Observational Cohort Study, 1993-2010. PLoS One. 2013;8(6).

16. Li Y, Ehiri J, Tang S, Li D, Bian Y, Lin H, et al. Factors associated with patient, and diagnostic delays in Chinese TB patients: a systematic review and meta-analysis. BMC Med. 2013;11:156.

17. Hussen A, Biadgilign S, Tessema F, Mohammed S, Deribe K, Deribew A. Treatment delay among pulmonary tuberculosis patients in pastoralist communities in Bale Zone, Southeast Ethiopia. BMC Res Notes. 2012;5:320.

18. Alavi SM, Sefidgaran G, Karami F. Comparative study on knowledge about tuberculosis in two cities with different tuberculosis epidemiological index in Khuzestan, 2004. Sci Med J. 2009;8(1):63-9.

19. Guneylioglu D, Yilmaz A, Bilgin S, Bayram U, Akkaya E. Factors affecting delays in diagnosis and treatment of pulmonary tuberculosis in a tertiary care hospital in Istanbul, Turkey. Med Sci Monit. 2004;10(2):CR62-7. 